Review Article

\title{
Efficacy of Praziquantel for the Treatment of Human Schistosomiasis in Ethiopia: A Systematic Review and Meta-Analysis
}

\author{
Tamirat Hailegebriel $\mathbb{D}^{1,},{ }^{1,2}$ Endalkachew Nibret $\mathbb{D}^{1},{ }^{1,2}$ and Abaineh Munshea $\mathbb{I D}^{1,2}$ \\ ${ }^{1}$ Department of Biology, College of Science, Bahir Dar University, Bahir Dar, Ethiopia \\ ${ }^{2}$ Biotechnology Research Institute (BRI), Bahir Dar University, Bahir Dar, Ethiopia \\ Correspondence should be addressed to Tamirat Hailegebriel; tamiratbdu@gmail.com
}

Received 11 August 2021; Accepted 4 December 2021; Published 20 December 2021

Academic Editor: Pedro P. Chieffi

Copyright (c) 2021 Tamirat Hailegebriel et al. This is an open access article distributed under the Creative Commons Attribution License, which permits unrestricted use, distribution, and reproduction in any medium, provided the original work is properly cited.

\begin{abstract}
Background. Schistosomiasis is one of the neglected tropical diseases causing a serious human health problem in Ethiopia. Praziquantel is the only drug that has been used for the treatment of human schistosomiasis in the country. In line with this, the efficacy of praziquantel has been evaluated in a few interventional studies in the country, but there is a lack in systematically gathered and analyzed information for policymakers. The aim of this systematic review and meta-analysis was to provide a summary of the efficacy of praziquantel for the treatment of human schistosomiasis in Ethiopia. Methods. We conducted a literature search from ScienceDirect, PubMed/Medlin, and Google Scholar databases. A total of 140 articles published in English from 1980 to June 2021 were accessed and 15 of them were eligible for this meta-analysis. The meta-analysis was conducted using Stata 14 software, "metan command." The heterogeneities among studies were evaluated using $I^{2}$ test. Results. A total of 140 articles were reviewed, but only 15 of them fulfilled the inclusion criteria. The polled cure rate of $40 \mathrm{mg} / \mathrm{kg}$ praziquantel was $89.2 \%$ (95\% CI: 85.4-93.1) and 93.6\% (95\% CI: 80.6-106) among Schistosoma mansoni and S. haematobium, respectively. Similarly, the mean egg reduction rates of $40 \mathrm{mg} / \mathrm{kg}$ praziquantel were $90.2 \%$ and $85 \%$ among S. mansoni and S. haematobium infected subjects, respectively. The common adverse events observed after receiving praziquantel include abdominal pain, vomiting, headache, diarrhea, and bloody stool. Conclusion. This systematic review and meta-analysis has indicated that praziquantel is still an appropriate drug for the treatment of human schistosomiasis in Ethiopia.
\end{abstract}

\section{Introduction}

Schistosomiasis is one of the neglected tropical diseases (NTD) which affects more than 230 million people in tropical and subtropical regions [1]. More than $90 \%$ of the cases are concentrated in African countries [2,3]. Schistosomiasis is responsible for about 4.5 million disabilityadjusted life years (DALYs) in endemic countries [4]. Human schistosomiasis is caused by six Schistosoma species. Among these, Schistosoma mansoni, S. haematobium, and S. japonicum are responsible for $99 \%$ of human schistosomiasis [5], of which the first two are the predominant and widely distributed species $[6,7]$, while $S$. japonicum is restricted in China, Indonesia, and parts of the Philippines [8].
In Ethiopia, estimates indicate that about 37.5 million people are living at risk of schistosomiasis, of which more than 5 million people are chronically infected [9]. Schistosomiasis is one of the major causes of outpatient morbidity in the country [10]. Schistosoma mansoni is the most common cause of human intestinal schistosomiasis followed by $S$. haematobium, which is a cause of urogenital schistosomiasis in the country. Although there is no nationwide survey, several epidemiological studies have indicated the existence of a moderate prevalence of schistosomiasis in the country. The prevalence of S. mansoni ranged from $10 \%$ to $92 \%$, while S. haematobium ranged from $5 \%$ to $58 \%$ in the country [11]. High prevalence of schistosomiasis has been reported among school-aged children in the country $[12,13]$. 
Although intensive research has been carried out on the development of a vaccine against schistosomiasis, there is no licensed vaccine yet. Thus, the available option is the use of praziquantel for the treatment of infected cases in many endemic countries since the 1970s. Besides praziquantel for treatment and a future vaccine for prevention, there are at least three other mainstems for tackling schistosomiasis: snail control, sanitation, and health education. Praziquantel is a relatively safe, well-absorbed, and effective oral drug that is active against all schistosome species [14]. The recommended dose is $40 \mathrm{mg} / \mathrm{kg}$ body weight and it is taken orally as a single dose $(40 \mathrm{mg} / \mathrm{kg})$, or this dose is divided over a day $(2 \times 20 \mathrm{mg} / \mathrm{kg}$ doses every 4 hours) [15] for the treatment of human schistosomiasis. Similarly, $60 \mathrm{mg} / \mathrm{kg}$ dose of praziquantel is also recommended for the treatment of human schistosomiasis for children [16, 17]. The drug usually acts within one hour of ingestion by paralyzing the worm and damaging the tegument. However, praziquantel has little or no effect on eggs and on immature worms [18]. In addition, repeated usage of praziquantel for the treatment of infected individuals and its mass drug administration among school children raise concerns about the efficacy of the drug in endemic countries. As a result, poor treatment outcomes of praziquantel were reported from several countries [19-21].

Praziquantel is widely used for the treatment of patients as well as mass drug administration (MDA) among schoolaged children in Ethiopia. Limited studies are available on the efficacy of praziquantel in the country. The standard approach to assess drug efficacy was by determining the cure rate $(\mathrm{CR})$ after administration of the standard dose of praziquantel. WHO has recently recommended egg reduction rate (ERR) as another approach to assess the efficacy of anthelminthic drugs [22]. CR is defined as the proportion of schistosome infected individuals who become parasitologically negative after administration of the standard dose (e.g., a single dose, $40 \mathrm{mg} / \mathrm{kg}$ ) of the drug. ERR is equal to 1(arithmetic mean of egg count after treatment divided by arithmetic mean egg count before treatment) expressed in percentage. Although quite limited and fragmented interventional studies are available in the country, to the best of our knowledge, there has not been any summarized data up to this point in time on the efficacy of praziquantel at the national level. Therefore, this systematic review and metaanalysis aimed to systematically summarize the existing fragmented efficacy studies in the country.

The PICOS (patients, interventions, comparisons, outcomes, and study design) framework used in this review is described as follows: P, schistosome infected individuals; I, administration of single-dose $40 \mathrm{mg} / \mathrm{kg}$ praziquantel; $\mathrm{C}$, no comparisons; O, schistosome infection cure-rate and egg reduction rate; and $\mathrm{S}$, observational studies.

\section{Methods}

2.1. Search Strategies. We conducted a literature search from online public databases, mainly from PubMed/Medline, Google Scholar, and ScienceDirect in June 2021. The selection was done using the following terms and Boolean operators: "Schistosomiasis" OR "praziquantel efficacy" OR
"S. mansoni" OR "S. haematobium" AND "Ethiopia." All articles published between 1980 and June 2021 in English were included in this meta-analysis. The systematic review and selection of relevant literature were conducted according to PRISMA (Preferred Reporting Items for Systematic Reviews and Meta-Analysis) guidelines [23] (Table S1).

2.2. Inclusion and Exclusion Criteria. Literatures published in the English language were extracted from online public databases. Only original articles published in peer review journals report the efficacy of praziquantel among Ethiopian populations. All articles that were interventional studies (observational studies) that administered single-dose praziquantel at $40 \mathrm{mg} / \mathrm{kg}$ body weight were included in the list of eligible articles. Articles that lacked information about cure rates or detailed information about study subjects and review papers and studies conducted on nonhuman subjects were excluded from this meta-analysis.

2.3. Data Extraction Protocol. The data extraction protocol was developed by TH and reviewed by EN and AM. The data extraction protocol consisted of the name of authors, year of publication, study region, sample size (population), population type (community or outpatient or school or preschool), age range, diagnostic methods, Schistosoma species targeted, cure rate praziquantel (number of cured/number of positive subjects), egg reduction rate, follow-up time, side effects of the praziquantel, and purpose of intervention (treatment or prevention).

2.4. Quality of Individual Study. The quality of individual studies was assessed using Quality Assessment Tools for interventional studies [24]. Individual studies were assigned a score of either No (0) or Yes (1) for the ten parameters that were formulated based on the objective of the review. The quality was determined by counting the number of Yes (1) scores in each of the ten parameters. The overall quality of the individual study was classified as low quality (scores of 1-4), moderate quality (scores of 5-7), and high quality (scores of 8-10).

2.5. Risk of Publication Bias. The risks of publication bias across studies were assessed using funnel plot symmetry (qualitative estimation) and Egger's regression test and Begg's rank correlation test (quantitative estimation).

2.6. Intervention and Outcomes. Schistosomiasis caused by S. mansoni and S. haematobium was included in this metaanalysis. The participants involved in the interventional studies were outpatients, school children, preschool children, and community-based studies of any age. The participants of all the interventional studies received the WHO recommended $40 \mathrm{mg} / \mathrm{kg}$ body weight single-dose praziquantel for the treatment of schistosomiasis. The outcome of the evaluation was a parasitological cure, which was defined 
as eggs-positive or eggs-negative or absence of symptoms associated with schistosomiasis.

2.7. Data Analysis. Cure rate was determined by dividing the number of cured individuals by the total positive subjects before treatment as indicated in each article. The confidence intervals for the cure rate were set at $95 \%$. The egg reduction rates were obtained from the articles and calculated as the arithmetic mean. All studies with a follow-up period of two to eight weeks were included in the meta-analysis. Subgroup analyses were conducted based on a region of study, nature of participants, Schistosoma species, and years of study. The random-effects model was used to combine the pooled cure rate due to the presence of heterogeneity among studies at 95\% CI. Egger's regression test and Begg's rank correlation test (quantitative) and funnel plot (qualitative) were used to assess the presence of publication bias. Metaregression analyses were performed to assess the sources of heterogeneity. The meta-analysis was performed using Stata software (version 14, STATA Corp, College Station, TX), where $p<0.05$ was considered as statistically significant.

\section{Results}

3.1. Selected Studies. From the total of 140 articles available online in public databases, 48 articles were excluded due to duplicates. Of the remaining 92 screened articles, 60 were excluded after we reviewed their titles and abstracts. The remaining 32 full-text articles were accessed for eligibility based on the inclusion criteria and information indicated in the data extraction protocol. As a result, 17 articles were further excluded in the data extraction process primarily due to the outcome of interest, having no cure rate, and lack of clear figures on the total number of study participants and treated cases. Only 15 articles [25-39] fulfilled the eligibility criteria and were included in this systematic review and meta-analysis (Figure 1).

3.2. Study Characteristics. The studies included in this metaanalysis were obtained from six regional states of Ethiopia, namely, Amhara, Oromia, Somali, South Nations and Nationalities Peoples (SNNP), Tigray regions, and Addis Ababa city administration. All eligible articles that were nonrandomized interventional studies were used in this metaanalysis. All interventional studies that used $40 \mathrm{mg} / \mathrm{kg}$ single-dose praziquantel with a follow-up period ranging from 2 to 8 weeks were included in the analysis. Detailed information about the characteristics of the articles included in this meta-analysis is shown in Table 1.

3.3. Risk of Bias within Studies. We assessed the quality of the individual study using Quality Assessment Tools for interventional studies. The quality score obtained from the individual study was moderate and high. The overall analysis showed that there was a low risk of publication bias within studies.
3.4. Pooled Efficacy of Praziquantel for the Treatment of Schistosomiasis. The efficacy of praziquantel was assessed using the cure rate and egg reduction rate among schistosome infected subjects. The cure rate of $40 \mathrm{mg} / \mathrm{kg}$ single-dose praziquantel was ranged from $73.6 \%$ to $100 \%$ among schistosome infected individuals in Ethiopia (Table 1). The pooled cure rate of a single dose of $40 \mathrm{mg} / \mathrm{kg}$ praziquantel was $89.8 \%$ (95\% CI: 86.2-93.5) for human schistosomiasis. The pooled cure rates of praziquantel were $89.2 \%$ (95\% CI: 85.4-93.1) and 93.6\% (95\% CI: 80.6-106) among S. mansoni and $S$. haematobium infected individuals, respectively (Figure 2). However, twelve of the total fourteen studies were carried out on S. mansoni, while only two studies were conducted on $S$. haematobium in the country.

Egg reduction rate was the second parameter used to assess the efficacy of praziquantel treatment. The ERR among $S$. mansoni infected individuals ranged from $79.5 \%$ to 99.6\%, while ERR was $85 \%$ among S. haematobium infected individuals. Unfortunately, 5 out of the 15 eligible articles (33.3\%) did not report ERR among treated individuals.

3.5. Publication Bias Across Studies. The funnel plot symmetry indicated that there was no publication bias across studies (Figure 3). Similarly, Egger's test $(p$ value $=0.069)$ and Begg's test $(p$ value $=0.138)$ confirmed the absence of publication bias among studies included in this metaanalysis.

3.6. Subgroup Analysis. The pooled cure rate of praziquantel was the highest, $96.6 \%$ (95\% CI: $88.5 \%-99.1 \%$ ) among preschool children followed by $95.9 \%$ (95\% CI: $86.3 \%-$ 98.9\%) among patients, $91.6 \%$ (95\% CI: $89.8 \%-93.5 \%)$ in community based studies, and $87.8 \%$ (95\% CI: $82.8 \%-$ $92.8 \%$ ) among schoolchildren (Figure 4). There was no significant difference in the efficacy of praziquantel against human schistosomiasis among regions in Ethiopia. The pooled cure rate of praziquantel treatment was $88.4 \%(95 \%$ CI: 84.8-91.8) in the Amhara region and 92.1\% (95\% CI: 80.8-100) in the Oromia region. The results of the efficacy study from Addis Ababa, Afar, Tigray, and SNNP regional states (single efficacy study from each region) are presented in Figure 5. We assessed the possibility of efficacy variation with respect to years of study (1980 to 2000 or 2001 to 2021). The pooled efficacy of praziquantel was $91.8 \%$ (1980 to 2000) and $89.0 \%$ (2001 to 2021) in the country (Figure 6).

3.7. Metaregression and Sensitivity Analysis. The result of this meta-analysis showed the presence of heterogeneity among studies $\left(I^{2}>90 \%\right)$. We performed a regression analysis to identify the sources of heterogeneity across studies included in this meta-analysis. The regression coefficient for several variables is as follows: region of study (1.007, 95\% CI: $0.978-1.038, p=0.596)$, nature of study participants (1.037, 95\% CI: 0.987-1.089, $p=0.138)$, year of publication (0.970, 95\% CI: 0.874-1.078, $p=0.548)$, and Schistosoma species (1.049, 95\% CI: 0.916-1.202, $p=0.459)$. However, these variables did not contribute to the observed 


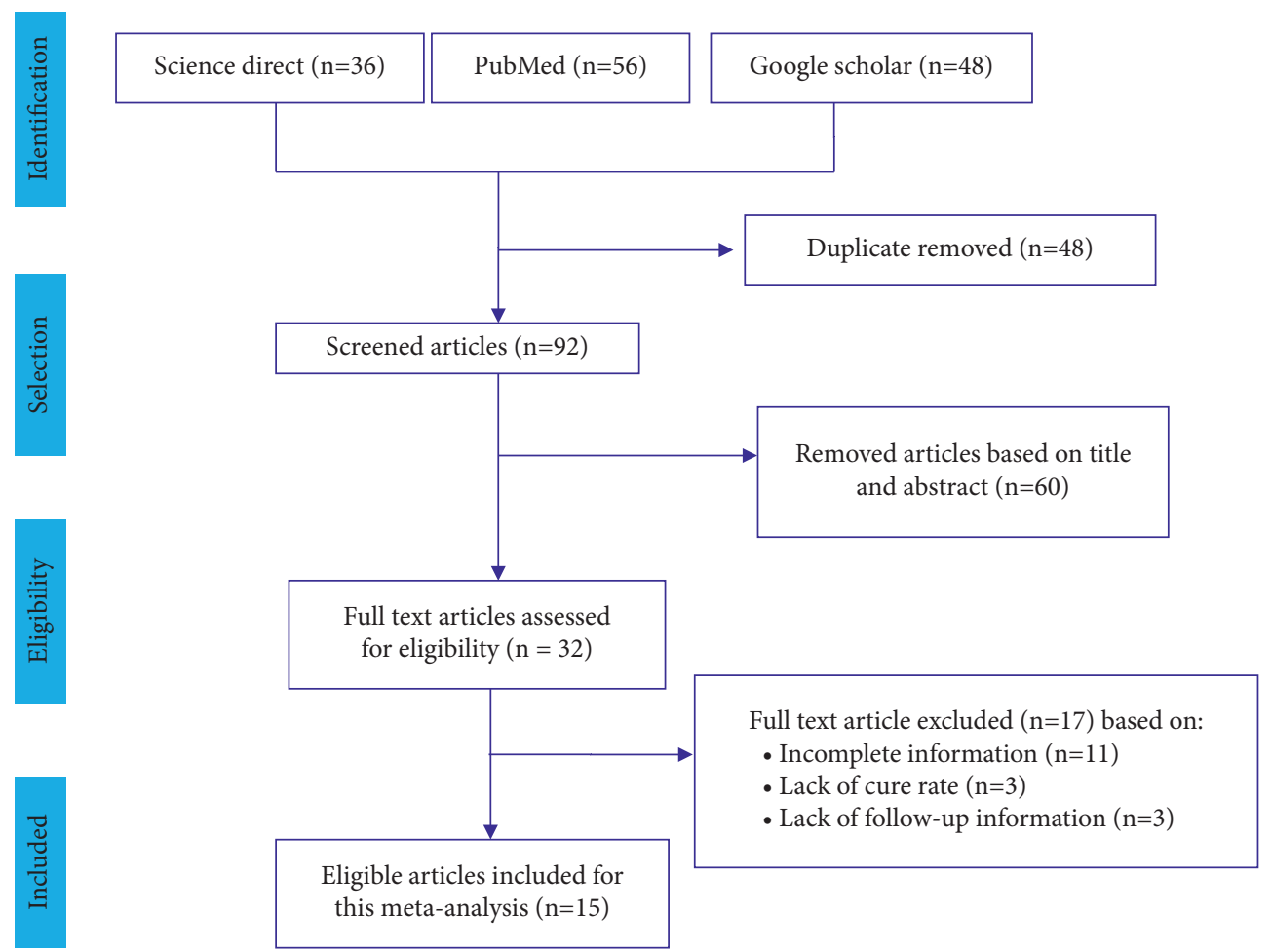

FIGURE 1: PRISMA flow diagram showing the selection process of articles used to determine the efficacy of praziquantel among the Ethiopian population.

heterogeneity. Sensitivity analysis was performed by excluding one interventional study at a time and calculates the pooled CR. The result of this analysis did not bring any significant change to the pooled CR.

3.8. Adverse Events. Adverse events were observed among $1079(88.7 \%)$ individuals who had taken praziquantel for the treatment of human schistosomiasis (Table 2). Adverse events of praziquantel were presented from 7 (50\%) of the interventional studies included in this review. The common adverse events include abdominal pain, headache and vomiting $[26,29,31-34,36]$, diarrhea $[26,29,32-34,36]$, bloody stool $[26,29,32,33,36]$, nausea [26, 29, 31-33], dizziness $[26,33,34,36]$, fatigue [29, 31-33], fever $[29,31-33]$, and drowsiness [29, 32, 33]. These signs and symptoms were observed for the first four hours after treatment and resolved shortly.

\section{Discussion}

Praziquantel is the only drug available for the treatment of human schistosomiasis in many endemic countries. Praziquantel has been used for the treatment of human schistosomiasis for more than forty years. Several interventional studies on the efficacy of the praziquantel are available in Ethiopia. Therefore, summarized data is needed to evaluate the overall efficacy of a single dose of $40 \mathrm{mg} / \mathrm{kg}$ praziquantel for the treatment of human schistosomiasis in the country.

This meta-analysis showed that a single dose of praziquantel has $89.8 \%$ cure rate for the treatment of human schistosomiasis in Ethiopia. In line with our findings, a higher cure rate of a single dose of $40 \mathrm{mg} / \mathrm{kg}$ praziquantel was reported in several countries [40-43]. In contrast, a lower cure rate of praziquantel was reported from a metaanalysis elsewhere $[44,45]$. The present study revealed that a single dose of $40 \mathrm{mg} / \mathrm{kg}$ praziquantel is effective against human schistosomiasis in Ethiopia. On the contrary, a repeated dose of $40 \mathrm{mg} / \mathrm{kg}$ praziquantel had a higher cure rate than a single dose of praziquantel $[17,46]$. Moreover, $60 \mathrm{mg} /$ $\mathrm{kg}$ praziquantel was more effective than the standard $40 \mathrm{mg} /$ $\mathrm{kg}$ single-dose praziquantel for the treatment of human schistosomiasis [47]. These variations of the efficacy of praziquantel might be associated with baseline infection intensity, nature of study population, sample size, and brand of the drug used.

The efficacy of praziquantel was slightly higher among S. haematobium infected patients than among S. mansoni infected cases. However, only two studies were conducted on the efficacy of praziquantel against $S$. haematobium from the total twelve studies included in this meta-analysis. Schistosoma haematobium is generally restricted in lowland (below 800 masl) areas [11, 31, 48, 49] and only a few studies are available in the country. Therefore, it is not wise to compare the efficacy of the drug against the two schistosome species based on the existing information. Similar to our finding, a higher cure rate of praziquantel was reported from preschool and schoolchildren infected with S. haematobium compared to those infected with $S$. mansoni $[44,47,50]$. In contrast, a higher cure rate of praziquantel was reported among individuals infected with $S$. mansoni than those infected with S. haematobium in Cameroon [51]. The difference in the 


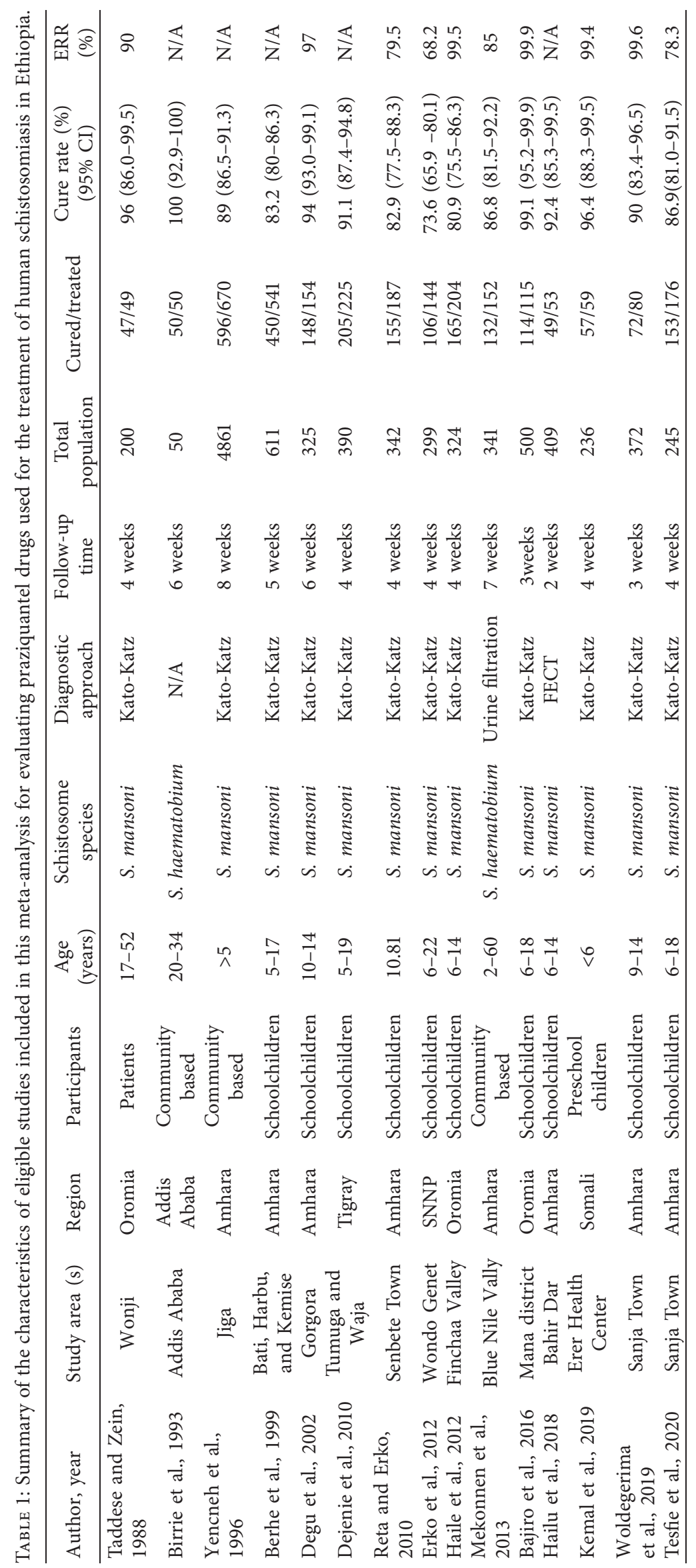




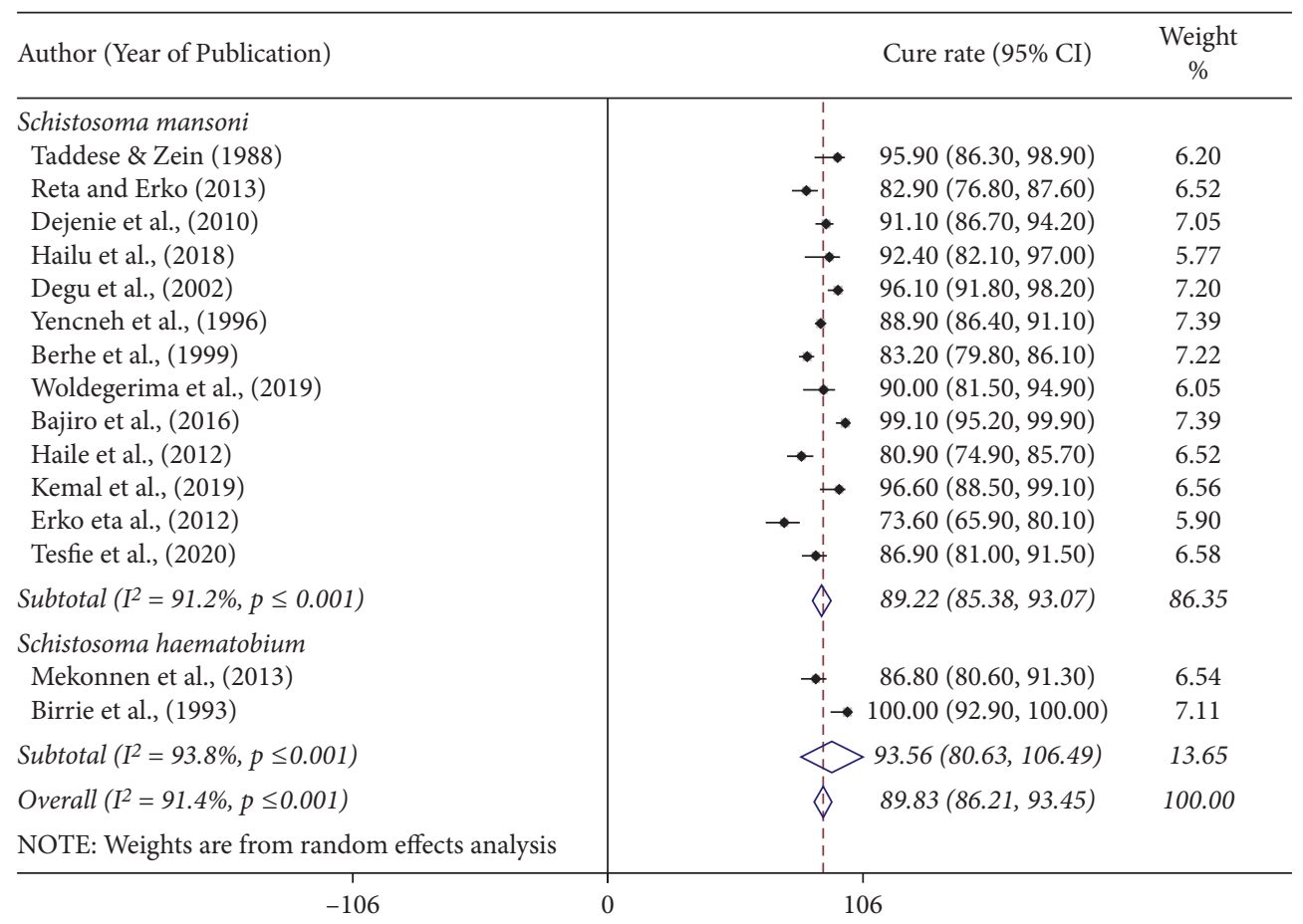

Figure 2: Forest plot showing the cure rate of praziquantel at $40 \mathrm{mg} / \mathrm{kg}$ for the treatment of human schistosomiasis based on Schistosoma species in Ethiopia.

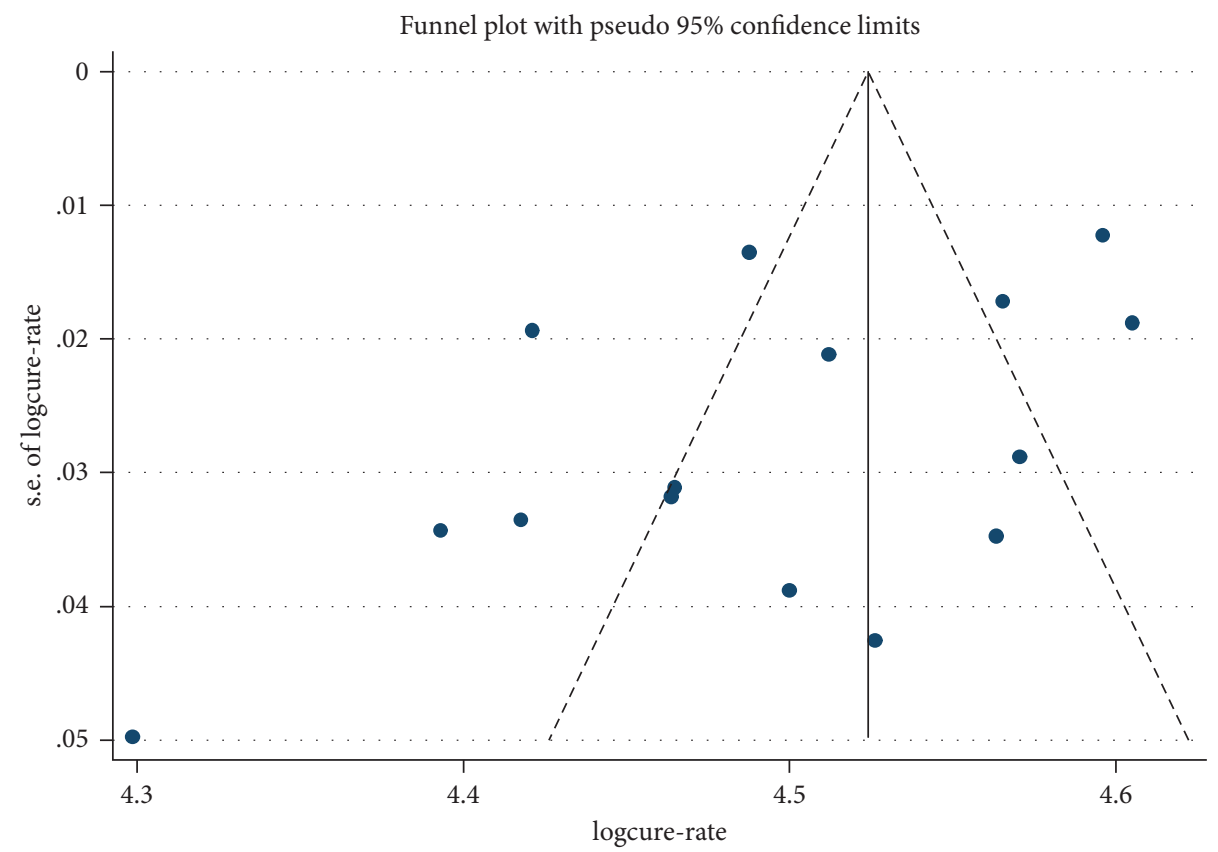

FIGURE 3: Egger's funnel plot indicating possible publication bias on the efficacy of $40 \mathrm{mg} / \mathrm{kg}$ praziquantel for the treatment of human schistosomiasis.

efficacy of praziquantel might be associated with the endemicity of the disease in the area, baseline infection intensity, age of study participants, and presence of immature stages in addition to the inherent biological difference of species.
The cure rate of a single dose of $40 \mathrm{mg} / \mathrm{kg}$ praziquantel was slightly lower in the Amhara region than in other regional states in Ethiopia. This might be associated with the high endemicity of the disease in the Amhara region. Several studies indicated high prevalence $(>83 \%)$ of S. mansoni in 


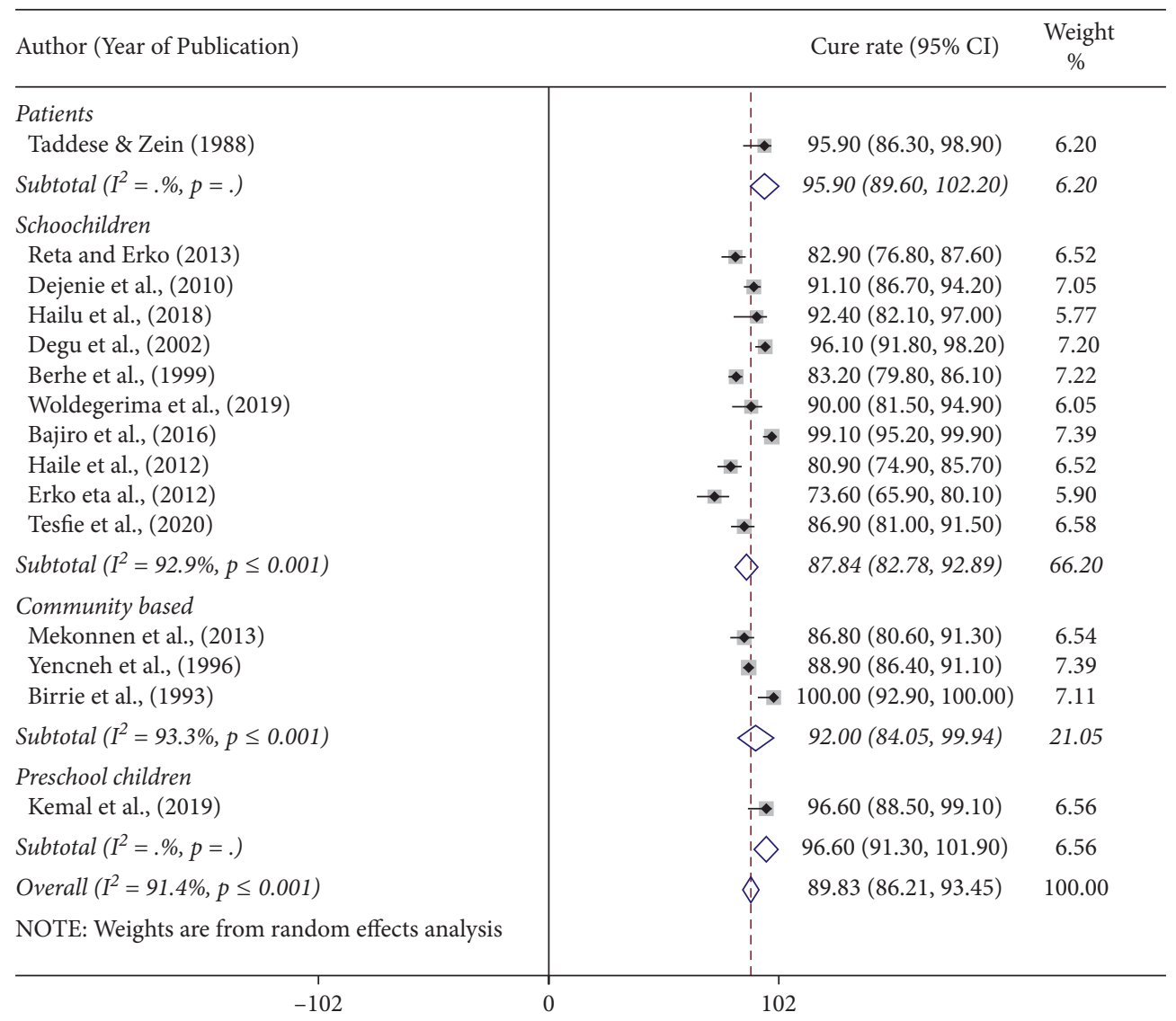

FIGURE 4: Forest plot showing the cure rate of praziquantel at $40 \mathrm{mg} / \mathrm{kg}$ for the treatment of human schistosomiasis based on the nature of study participants in Ethiopia.

the region $[12,13,38]$. The high prevalence and intensity of infection may contribute to the slightly lower efficacy of praziquantel in the region. In line with the high infection intensity, there may be large number of immature worms, which is insensitive for the drug that contributes to lower efficacy in the region. Moreover, 8 (53.3\%) of the interventional studies included in this meta-analysis were derived from the Amhara region. There were only four studies reported from Tigray, SNNP, Somalia, and Addis Ababa city administrations (one study from each regional state). As the number of studies increased, there might be a variation in their methodology, follow-up time, level of endemicity, nature of the study population, and sample size. These variations may lead to differences in the efficacy of praziquantel among interventional studies which might contribute to the slightly reduced cure rate in the region.

The efficacies of praziquantel were almost similar in all types of study participants (patients, schoolchildren, preschool children, and the community). The efficacy of praziquantel was slightly higher among preschool children than among schoolchildren as reported elsewhere [44]. In this meta-analysis, only one study reported the efficacy of praziquantel from preschool children in Ethiopia. It might not be good to compare the efficacy of praziquantel between schoolchildren and preschool children. Similarly, we compared the cure rate of praziquantel from 1980 to 2000 and from 2001 to 2021 to assess the possibility of variation in response rate due to frequent exposure. The result showed that the cure rate is slightly lower in recent years than in studies reported before 2000 . However, the difference is not statistically significant. This shows the absence of drug failure and/or resistance against praziquantel, suggesting that the drug is still effective in the treatment of schistosomiasis in Ethiopia.

The study showed high heterogeneity $\left(I^{2}=91.4 \%\right)$ across studies. We conducted a metaregression analysis to assess the source of heterogeneity by considering region of study, nature of study participants, year of study, and Schistosoma species. All these did not contribute to the observed heterogeneity. Other conditions such as sample size, infection intensity, and specific intervention time and area might contribute to the observed heterogeneity across studies.

Egg reduction rate was the second recommended option to assess the efficacy of anthelminthic drugs in endemic countries [22]. The egg reduction rate after praziquantel administration reported among studies included in this meta-analysis ranged from $68.2 \%$ to $99.6 \%$. The pooled egg reduction rate was $89.6 \%$ among studies that reported ERR. Praziquantel contributes to more than $90 \%$ of ERR of schistosome infection as reported elsewhere [41, 45, 52]. Anthelminthic drugs that reduce the infection intensity at least by $90 \%$ are accepted and are recommended to continue 


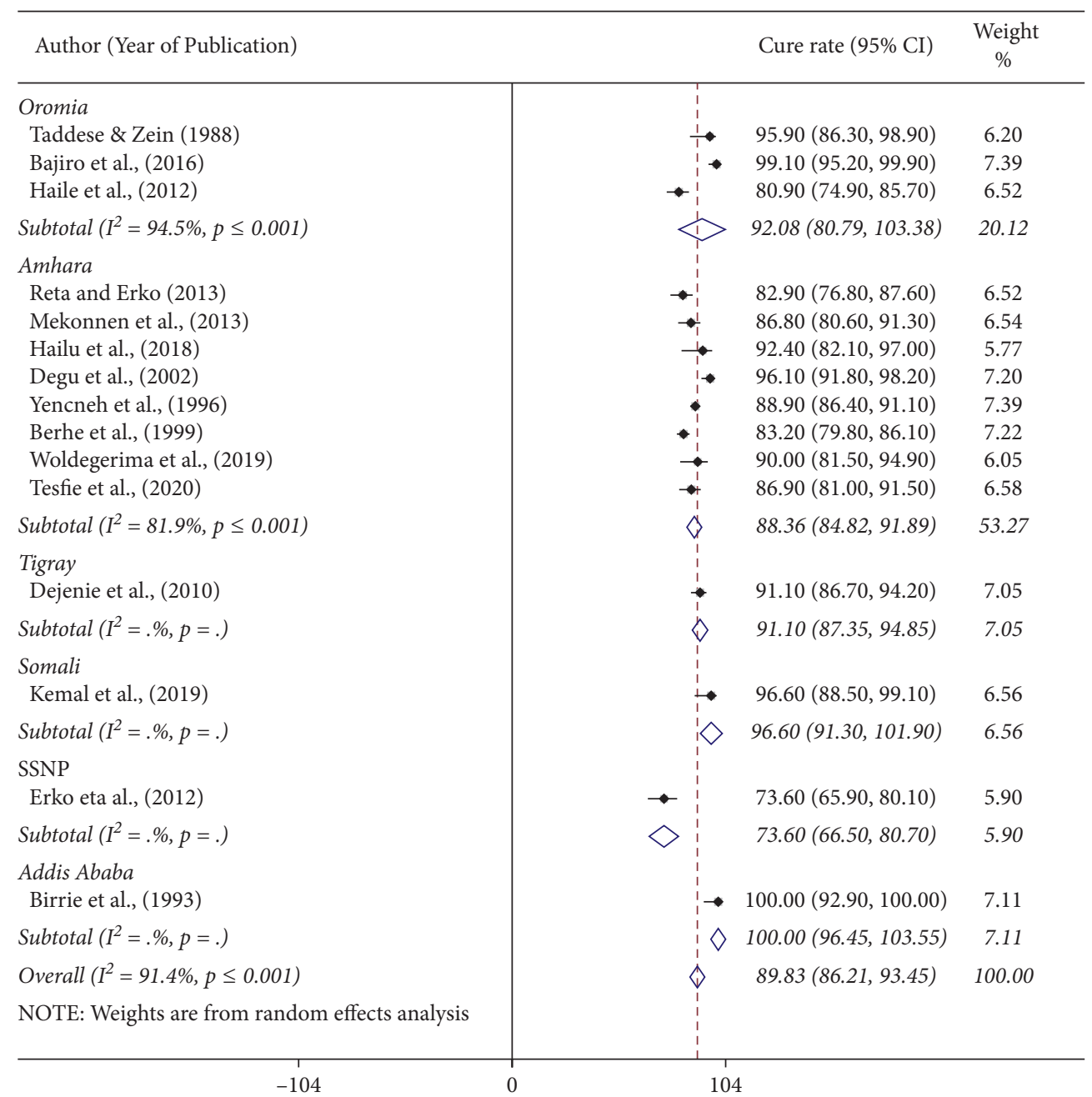

FigURE 5: Forest plot showing the cure rate of praziquantel at $40 \mathrm{mg} / \mathrm{kg}$ for the treatment of human schistosomiasis in Ethiopia by region of study.

for the treatment of human schistosomiasis [22]. The studies included in this meta-analysis used the standard $40 \mathrm{mg} / \mathrm{kg}$ single-dose praziquantel. Studies showed that a double-dose arm of $40 \mathrm{mg} / \mathrm{kg}$ of praziquantel resulted in a higher ERR compared to the standard single-dose arm $[17,46]$.

About $7 / 14$ (50\%) of the interventional studies reported the presence of adverse events of praziquantel within 4 hours after drug administration, but all adverse events were subsided within a short period of time. The most common adverse events including abdominal pain/discomfort/cramp, headache, and vomiting followed by diarrhea, nausea, itching, bloody stool, fever, fatigue, and dizziness were reported in at least four studies from the total seven studies that reported adverse events of praziquantel. Similar adverse events of praziquantel were reported in systematic review and meta-analysis from schistosomiasis endemic countries $[44,45]$. The adverse events might be associated with the host immune response to the parasite antigens released from the dead parasites. In addition, the intensity and location of the infection may also contribute to the adverse side effects.

About $89 \%$ of the treated individuals showed one or more adverse events of praziquantel observed in study subjects included in this review. Similar high adverse events of praziquantel were reported from Brazil [53] and Uganda [54]. In contrast to our findings, a lower adverse event of praziquantel was reported elsewhere [43, 45, 50]. These differences might be associated with the difference in infection intensity, parasite, or host factors.

\section{Limitation of the Study}

This meta-analysis summarized the praziquantel efficacy studies carried out in the country and produced pooled cure rates and egg reduction rates. Nevertheless, it has some limitations. First, the studies included in this meta-analysis lack a control group or individuals taking a placebo during the intervention time. This makes it is impossible to calculate risk ratio $(\mathrm{RR})$ or relative risk. Therefore, we were forced to use the cure rate rather than the risk ratio. Second, the studies eligible for this meta-analysis used different parasitological methods (Kato-Katz, urine filtration, and formolether concentration). These methods have different sensitivity and specificity that may affect the outcome of praziquantel efficacy. It is known that Kato-Katz and urine 


\begin{tabular}{|c|c|c|}
\hline Author (Year of Publication) & Cure rate $(95 \% \mathrm{CI})$ & $\begin{array}{c}\text { Weight } \\
\%\end{array}$ \\
\hline \multicolumn{3}{|l|}{1980 to 2000} \\
\hline Taddese \& Zein (1988) & - $95.90(86.30,98.90)$ & 6.20 \\
\hline Yencneh et al., (1996) & $88.90(86.40,91.10)$ & 7.39 \\
\hline Berhe et al., (1999) & $83.20(79.80,86.10)$ & 7.22 \\
\hline Birrie et al., (1993) & $\rightarrow 100.00(92.90,100.00)$ & 7.11 \\
\hline Subtotal $\left(I^{2}=94.3 \%, p \leq 0.001\right)$ & $91.83(84.60,99.05)$ & 27.92 \\
\hline \multicolumn{3}{|l|}{2001 to 2020} \\
\hline Reta and Erko (2013) & $82.90(76.80,87.60)$ & 6.52 \\
\hline Mekonnen et al., (2013) & $86.80(80.60,91.30)$ & 6.54 \\
\hline Dejenie et al., (2010) & $91.10(86.70,94.20)$ & 7.05 \\
\hline Hailu et al., (2018) & $92.40(82.10,97.00)$ & 5.77 \\
\hline Degu et al., (2002) & $\rightarrow \quad 96.10(91.80,98.20)$ & 7.20 \\
\hline Woldegerima et al., (2019) & $\stackrel{\mathrm{l}}{\rightarrow} \quad 90.00(81.50,94.90)$ & 6.05 \\
\hline Bajiro et al., (2016) & $\rightarrow 99.10(95.20,99.90)$ & 7.39 \\
\hline Haile et al., (2012) & $80.90(74.90,85.70)$ & 6.52 \\
\hline Kemal et al., (2019) & $96.60(88.50,99.10)$ & 6.56 \\
\hline Erko eta al., (2012) & $\rightarrow-73.60(65.90,80.10)$ & 5.90 \\
\hline Tesfie et al., (2020) & $86.90(81.00,91.50)$ & 6.58 \\
\hline Subtotal $\left(I^{2}=90.4 \%, p \leq 0.001\right)$ & $89.03(84.56,93.51)$ & 72.08 \\
\hline Overall $\left(I^{2}=91.4 \%, p \leq 0.001\right)$ & $89.83(86.21,93.45)$ & 100.00 \\
\hline \multicolumn{3}{|l|}{ NOTE: Weights are from random effects analysis } \\
\hline-100 & 100 & \\
\hline
\end{tabular}

FIGURE 6: Forest plot showing the cure rate of praziquantel at $40 \mathrm{mg} / \mathrm{kg}$ for the treatment of human schistosomiasis based on years of studies in Ethiopia.

TABLE 2: Adverse side effects observed among individuals treated with $40 \mathrm{mg} / \mathrm{kg}$ praziquantel in Ethiopia.

\begin{tabular}{|c|c|c|c|c|c|c|}
\hline \multirow{2}{*}{ Adverse events reported } & \multirow{2}{*}{ No. of studies } & \multirow{2}{*}{ Patients treated } & \multirow{2}{*}{ Treated patients showing AEs } & \multirow{2}{*}{ Incidence (\%) } & \multicolumn{2}{|c|}{$95 \% \mathrm{CI}$} \\
\hline & & & & & Lower limit & Upper limit \\
\hline Abdominal pain/cramp & 7 & 1217 & 842 & 69.2 & 66.5 & 71.8 \\
\hline Diarrhea & 6 & 1158 & 419 & 36.2 & 33.4 & 39.0 \\
\hline Headache & 7 & 1217 & 345 & 28.3 & 25.8 & 30.9 \\
\hline Nausea & 5 & 1066 & 317 & 29.7 & 27.0 & 32.6 \\
\hline Vomiting & 7 & 1217 & 305 & 25.1 & 22.6 & 27.6 \\
\hline Dizziness & 4 & 839 & 237 & 28.2 & 25.2 & 41.4 \\
\hline Bloody stool & 5 & 1108 & 184 & 16.6 & 14.5 & 18.9 \\
\hline Body weakness & 2 & 724 & 157 & 21.7 & 18.7 & 24.9 \\
\hline Fever & 4 & 537 & 138 & 25.7 & 22.1 & 29.6 \\
\hline Fatigue & 4 & 537 & 126 & 23.5 & 19.9 & 27.3 \\
\hline Straining & 2 & 283 & 116 & 40.9 & 35.2 & 46.9 \\
\hline Itching & 5 & 629 & 89 & 14.1 & 11.5 & 17.1 \\
\hline Drowsiness & 3 & 478 & 88 & 18.4 & 15.0 & 22.2 \\
\hline Haematuria & 1 & 65 & 65 & 98.5 & 91.7 & 100.0 \\
\hline Epigastric pain & 2 & 694 & 63 & 9.1 & 7.0 & 11.5 \\
\hline Dysuria & 2 & 283 & 58 & 20.5 & 15.9 & 25.7 \\
\hline Stomachache & 1 & 59 & 10 & 16.9 & 8.4 & 28.9 \\
\hline Dermatological code & 1 & 101 & 9 & 8.9 & 4.2 & 16.2 \\
\hline Sweating & 1 & 59 & 4 & 6.8 & 1.9 & 16.5 \\
\hline Body/skin rash & 1 & 195 & 3 & 1.5 & 0.3 & 4.4 \\
\hline Swelling & 1 & 195 & 3 & 1.3 & 0.3 & 4.4 \\
\hline Any one of these AEs & 7 & 1217 & 1079 & 88.7 & 86.7 & 90.4 \\
\hline
\end{tabular}

filtration methods are needed for the diagnosis of S. mansoni and S. haematobium infection, respectively. Third, egg reduction rate was not available in all articles included in this meta-analysis. Fourth, the adverse side effects of praziquantel were not reported from all eligible articles for this study. 


\section{Conclusion}

This systematic review and meta-analysis revealed that praziquantel is still an effective drug for the treatment of human schistosomiasis in Ethiopia. A single dose of $40 \mathrm{mg} / \mathrm{kg}$ praziquantel administration resulted in $>90 \%$ in both cure and egg reduction rates in Ethiopia. Mild and transit adverse effects including abdominal pain/discomfort, headache, vomiting, diarrhea, bloody stool, nausea, and itching were consistently observed among individuals after receiving the treatment but resolved within 24 hours. This meta-analysis supports the use of a single dose of $40 \mathrm{mg} / \mathrm{kg}$ praziquantel for the treatment of patients as well as for mass drug administration. Praziquantel treatment should be supported by environmental sanitation and proper health education for effective control and elimination of human schistosomiasis in Ethiopia.

\section{Abbreviations}

$\begin{array}{ll}\text { AEs: } & \text { Adverse events } \\ \text { CI: } & \text { Confidence interval } \\ \text { CR: } & \text { Cure rate } \\ \text { DALYs: } & \text { Disability-adjusted life years } \\ \text { ERR: } & \text { Egg reduction rate } \\ \text { FECT: } & \text { Formalin-ether concentration technique } \\ \text { I }^{2}: & \text { Inverse Variance Index } \\ \text { MDA: } & \text { Mass drug administration } \\ \text { NTD: } & \text { Neglected tropical diseases } \\ \text { PRISMA: } & \text { Preferred Reporting Items for Systematic } \\ & \text { Reviews and Meta-Analysis } \\ \text { RR: } & \text { Risk ratio } \\ \text { SNNP: } & \text { South Nations and Nationalities Peoples } \\ \text { WHO: } & \text { World Health Organization. }\end{array}$

\section{Data Availability}

All data are included within the manuscript.

\section{Conflicts of Interest}

The authors declare that there are no conflicts of interest.

\section{Authors' Contributions}

Tamirat Hailegebriel contributed to the design and conduct of the review, data analysis, interpretation of the findings, and drafting of the manuscript. Endalkachew Nibret and Abaineh Munshea contributed to drafting, reviewing, and editing the manuscript.

\section{Acknowledgments}

The authors would like to thank all of the authors of the eligible studies included in this systematic review and metaanalysis.

\section{Supplementary Materials}

Table S1: PRISMA checklist: the Preferred Reporting Items for Systematic Reviews and Meta-Analysis that indicates how the selection of relevant literatures and the systematic review were conducted. (Supplementary Materials)

\section{References}

[1] WHO, Schistosomiasis, WHO, Geneva, Switzerland, 2020, https://www.who.int/news-room/fact-sheets/detail/ schistosomiasis.

[2] P. Steinmann, J. Keiser, R. Bos, M. Tanner, and J. Utzinger, "Schistosomiasis and water resources development: systematic review, meta-analysis, and estimates of people at risk," The Lancet Infectious Diseases, vol. 6, no. 7, pp. 411-425, 2006.

[3] M. A. Barry, G. G. Simon, N. Mistry, and P. J. Hotez, "Global trends in neglected tropical disease control and elimination: impact on child health," Archives of Disease in Childhood, vol. 98, no. 8, pp. 635-641, 2013.

[4] C. H. King, K. Dickman, and D. J. Tisch, "Reassessment of the cost of chronic helmintic infection: a meta-analysis of disability-related outcomes in endemic schistosomiasis," The Lancet, vol. 365, no. 9470, pp. 1561-1569, 2005.

[5] A. Pinto-Almeida, T. Mendes, R. N. de Oliveira et al., "Morphological characteristics of schistosoma mansoni PZQresistant and -susceptible strains are different in presence of praziquantel," Frontiers in Microbiology, vol. 7, no. 594, 2016.

[6] M. Merrifield, P. J. Hotez, C. M. Beaumier et al., "Advancing a vaccine to prevent human schistosomiasis," Vaccine, vol. 34, no. 26, pp. 2988-2991, 2016.

[7] D. Rollinson, J. R. Stothard, and V. R. Southgate, "Interactions between intermediate snail hosts of the genus Bulinus and schistosomes of the Schistosoma haematobium group," Parasitology, vol. 123, no. 7, pp. 245-260, 2001.

[8] C. Gordon, J. Kurscheid, G. Williams et al., "Asian schistosomiasis: current status and prospects for control leading to elimination," Tropical Medicine and Infectious Disease, vol. 4, no. 1, p. 40, 2019.

[9] N. Negussu, B. Mengistu, B. Kebede et al., "Ethiopia schistosomiasis and soil-transmitted helminthes control programme: progress and prospects," Ethiopian Medical Journal, vol. 55, no. 1, pp. 75-80, 2017.

[10] T. Hailu and M. Yimer, "Prevalence of Schistosoma mansoni and geo-helminthic infections among patients examined at workemeda health center, northwest Ethiopia," Journal of Parasitology and Vector Biology, vol. 6, no. 5, pp. 75-79, 2014.

[11] K. Helmut, L. C. Tsong, H. Birrie, T. Ayele, S. Tedla, and F. Tsegay, "Schistosomiasis in Ethiopia," Social Science \& Medicine, vol. 26, no. 8, pp. 803-827, 1988.

[12] L. Worku, D. Damte, M. Endris, H. Tesfa, and M. Aemero, "Schistosoma mansoni infection and associated determinant factors among school children in sanja town, northwest Ethiopia," Journal of Parasitology Research, vol. 2014, Article ID 792536, 2014.

[13] G. Alebie, B. Erko, M. Aemero, and B. Petros, "Epidemiological study on Schistosoma mansoni infection in Sanja area, Amhara region, Ethiopia," Parasites \& Vectors, vol. 7, no. 1, p. 15, 2014.

[14] M. J. Doenhoff, D. Cioli, and J. Utzinger, "Praziquantel: mechanisms of action, resistance and new derivatives for schistosomiasis," Current Opinion in Infectious Diseases, vol. 21, no. 6, pp. 659-667, 2008. 
[15] B. Gryseels, K. Polman, J. Clerinx, and L. Kestens, "Human schistosomiasis," The Lancet, vol. 368, no. 9541, pp. 1106-1118, 2006.

[16] WHO, Prevention and Control of Schistosomiasis and SoilTransmitted Helminthiasis, WHO, Geneva, Switzerland, 2002.

[17] D. Z. Munisi, J. Buza, E. A. Mpolya, T. Angelo, and S. M. Kinung'hi, "The efficacy of single-dose versus doubledose praziquantel treatments onSchistosoma mansoniInfections: its implication on undernutrition and anaemia among primary schoolchildren in two on-shore communities, Northwestern Tanzania," BioMed Research International, vol. 2017, pp. 1-13, 2017.

[18] D. P. McManus, D. W. Dunne, M. Sacko, J. Utzinger, B. J. Vennervald, and X.-N. Zhou, "Schistosomiasis," Nature Reviews Disease Primers, vol. 4, no. 1, p. 13, 2018.

[19] I. M. d. Silva, R. Thiengo, M. J. Conceição et al., "Therapeutic failure of praziquantel in the treatment of Schistosoma haematobium infection in Brazilians returning from Africa," Memórias do Instituto Oswaldo Cruz, vol. 100, no. 4, pp. 445-449, 2005.

[20] S. D. Melman, M. L. Steinauer, C. Cunningham et al., "Reduced susceptibility to praziquantel among naturally occurring Kenyan isolates of schistosoma mansoni," PLoS Neglected Tropical Diseases, vol. 3, no. 8, Article ID e504, 2009.

[21] M. J. Doenhoff, J. R. Kusel, G. C. Coles, and D. Cioli, "Resistance of Schistosoma mansoni to praziquantel: is there a problem?" Transactions of the Royal Society of Tropical Medicine and Hygiene, vol. 96, no. 5, pp. 465-469, 2002.

[22] WHO, Assessing the Efficacy of Anthelminthic Drugs against Schistosomiasis and Soil-Transmitted Helminthiases, World Health Organization, Geneva, Switzerland, 2013, https://apps. who.int/iris/handle/10665/79019.

[23] D. Moher, A. Liberati, J. Tetzlaff, and D. G. Altman, "Preferred reporting items for systematic reviews and meta-analyses: the PRISMA statement," BMJ, vol. 339, no. 1, Article ID b2535, 2009.

[24] NIH Study, Quality Assessment Tools, https://www.nhlbi.nih. gov/health-topics/study-quality-assessment-tools, 2020.

[25] B. Nega, G. G. Svein, A. Fekadu, B. Hailu, M. Girmay, and G. Teferi, "Praziquantel side effects and efficacy related to Schistosoma mansoni egg loads and morbidity in primary school children in north-east Ethiopia," Acta Tropica, vol. 72, pp. 53-63, 1999.

[26] G. Degu, G. Mengistu, and J. Jones, "Praziquantel efficacy against schistosomiasis mansoni in schoolchildren in northwest Ethiopia," Transactions of the Royal Society of Tropical Medicine and Hygiene, vol. 96, no. 4, pp. 444-445, 2002.

[27] T. Dejenie, T. Asmelash, and M. Abdelkadir, "Efficacy of praziquantel in treating schistosoma mansoni infected school children in tumuga and waja, North Ethiopia," Momona Ethiopian Journal of Science, vol. 2, no. 2, pp. 3-11, 2010.

[28] E. Berhanu, A. Degarege, K. Tadesse, A. Mathiwos, and M. Legesse, "Efficacy and side effects of praziquantel in the treatment of Schistosoma mansoni in schoolchildren in shesha Kekele elementary school, wondo Genet, southern Ethiopia," Asian Pacific Journal of Tropical Biomedicine, vol. 2, no. 3, pp. 235-239, 2012.

[29] S. Haile, L. Golassa, and Z. Mekonnen, "Prevalence of Schistosoma mansoni and effectiveness of Praziquantel in school children in Finchaa valley, Ethiopia," Journal of Parasitology and Vector Biology, vol. 4, no. 3, pp. 25-30, 2012.

[30] M. Kemal, G. Tadesse, A. Esmael, S. M. Abay, and T. Kebede, "Schistosoma mansoni infection among preschool age children attending Erer Health Center, Ethiopia and the response rate to praziquantel," BMC Research Notes, vol. 12, no. 1, p. 211, 2019.

[31] M. A. Asaye, M. Legesse, M. Belay, K. Tadesse, W. Torben, and Z. Teklemariam, "Efficacy of praziquantel against Schistosoma haematobium in Dulshatalo village, western Ethiopia," BMC Research Notes, vol. 6, p. 392, 2013.

[32] B. Reta and B. Erko, "Efficacy and side effects of praziquantel in the treatment forSchistosoma mansoniinfection in school children in Senbete Town, northeastern Ethiopia," Tropical Medicine and International Health, vol. 18, no. 11, pp. 1338-1343, 2013.

[33] K. Taddese and Z. A. Zein, "Comparison between the efficacy of oxamniquine and praziquantel in the treatment ofSchistosoma mansoniinfections on a sugar estate in Ethiopia," Annals of Tropical Medicine and Parasitology, vol. 82, no. 2, pp. 175-180, 1988.

[34] E. Woldegerima, A. G. Bayih, Y. Tegegne, M. Aemero, and A. J. Zeleke, "Prevalence and reinfection rates of Schistosoma mansoni and praziquantel efficacy against the parasite among primary school children in sanja town, northwest Ethiopia," Journal of Parasitology Research, vol. 2019, Article ID 3697216, 2019.

[35] H. Yencneh, F. Abebe, M. Ameha, B. Hailu, and A. Teklemariam, "Praziquantel in the control of Schistosoma mansoni infection in Jiga, northwestern Ethiopia," The Ethiopian Journal of Health Development, vol. 10, no. 2, pp. 105-110, 1996.

[36] T. Hailu, B. Abera, W. Mulu, M. Alemu, E. Yizengaw, and A. Genanew, "Efficacy of single dose albendazole and praziquantel drugs among helminth-infected school children at Rural Bahir Dar, Northwest Ethiopia," Tropical Doctor, vol. 48, no. 4, pp. 270-272, 2018.

[37] H. Birrie, N. Berhe, S. Tedla, and N. Gemeda, "Schistosoma haematobium infection among Ethiopiam prisoners of war (1977-1988) returning from Somalia," Ethiopian Medical Journal, vol. 31, no. 4, pp. 259-264, 1993.

[38] A. Tesfie, G. Getnet, A. Abere et al., "Praziquantel is an effective drug for the treatment of Schistosoma mansoni infection among school-aged children in Northwest Ethiopia," Tropical Medicine and Health, vol. 48, no. 1, p. 28, 2020.

[39] M. Bajiro, D. Dana, M. Ayana et al., "Prevalence of Schistosoma mansoni infection and the therapeutic efficacy of praziquantel among school children in Manna District, Jimma Zone, southwest Ethiopia," Parasites \& Vectors, vol. 9, no. 1, p. 560, 2016.

[40] A. M. Ahmed, H. Abbas, F. A. Mansour, G. I. Gasim, and I. Adam, "Schistosoma haematobium infections among schoolchildren in central Sudan one year after treatment with praziquantel," Parasites \& Vectors, vol. 5, no. 1, p. 1, 2012.

[41] J. T. Coulibaly, Y. K. N'Gbesso, S. Knopp, J. Keiser, E. K. N'Goran, and J. Utzinger, "Efficacy and safety of praziquantel in preschool-aged children in an area Co-endemic for schistosoma mansoni and S. Haematobium," PLoS Neglected Tropical Diseases, vol. 6, no. 12, Article ID e1917, 2012.

[42] M. A. Amin, M. Swar, M. Kardaman, D. Elhussein, G. Nouman, and A. Mahmoud, "Treatment of pre-school children under 6 years of age for schistosomiasis: safety, efficacy and acceptability of praziquantel," Sudan Journal of Medical Sciences, vol. 7, pp. 67-76, 2012.

[43] V. Y. Belizario Jr, M. L. E. Amarillo, R. M. Martinez, A. O. Mallari, and C. M. C. Tai, "Efficacy and safety of $40 \mathrm{mg} /$ $\mathrm{kg}$ and $60 \mathrm{mg} / \mathrm{kg}$ single doses of praziquantel in the treatment 
of schistosomiasis," Journal of Pediatric Infectious Diseases, vol. 3, no. 1, pp. 27-34, 2008.

[44] R. Liu, H.-F. Dong, Y. Guo, Q.-P. Zhao, and M.-S. Jiang, "Efficacy of praziquantel and artemisinin derivatives for the treatment and prevention of human schistosomiasis: a systematic review and meta-analysis," Parasites \& Vectors, vol. 4, no. 1, p. 201, 2011.

[45] J. Zwang and P. L. Olliaro, "Clinical efficacy and tolerability of praziquantel for intestinal and urinary schistosomiasis-a meta-analysis of comparative and non-comparative clinical trials," PLoS Neglected Tropical Diseases, vol. 8, no. 11, Article ID e3286, 2014.

[46] C. H. King, S. K. Olbrych, M. Soon, M. E. Singer, J. Carter, and D. G. Colley, "Utility of repeated praziquantel dosing in the treatment of schistosomiasis in high-risk communities in Africa: a systematic review," PLoS Neglected Tropical Diseases, vol. 5, no. 9, Article ID e1321, 2011.

[47] M. Kabuyaya, M. J. Chimbari, and S. Mukaratirwa, "Efficacy of praziquantel treatment regimens in pre-school and school aged children infected with schistosomiasis in sub-Saharan Africa: a systematic review," Infectious Diseases of Poverty, vol. 7 , no. 1, p. 73, 2018 .

[48] K. Deribew, Z. Tekeste, and B. Petros, "Urinary schistosomiasis and malaria associated anemia in Ethiopia," Asian Pacific Journal of Tropical Biomedicine, vol. 3, no. 4, pp. 307-310, 2013.

[49] G. de Sole, A. Lemma, and B. Mazengia, "Schistosoma haematobium in the wabi shebelle valley of Ethiopia," The American Journal of Tropical Medicine and Hygiene, vol. 27, no. 5, pp. 928-930, 1978.

[50] J. Zwang and P. Olliaro, "Efficacy and safety of praziquantel 40 $\mathrm{mg} / \mathrm{kg}$ in preschool-aged and school-aged children: a metaanalysis," Parasites \& Vectors, vol. 10, no. 1, p. 47, 2017.

[51] L.-A. T. Tchuenté, S. C. Momo, and J. R. Stothard, D. Rollinson, Efficacy of praziquantel and reinfection patterns in single and mixed infection foci for intestinal and urogenital schistosomiasis in Cameroon," Acta Tropica, vol. 128, no. 2, pp. 275-283, 2013.

[52] J. C. Sousa-Figueiredo, M. Betson, A. Atuhaire et al., "Performance and safety of praziquantel for treatment of intestinal schistosomiasis in infants and preschool children," PLoS Neglected Tropical Diseases, vol. 6, no. 10, Article ID e1864, 2012.

[53] N. Katz, R. S. Rocha, and A. Chaves, "Preliminary trials with praziquantel in human infection due to Schistosoma mansoni," Bulletin of the World Health Organization, vol. 57, pp. 781-784, 1979.

[54] N. B. Kabatereine, J. Kemijumbi, J. H. Ouma et al., "Efficacy and side effects of praziquantel treatment in a highly endemicSchistosoma mansoni focus at Lake Albert, Uganda," Transactions of the Royal Society of Tropical Medicine and Hygiene, vol. 97, no. 5, pp. 599-603, 2003. 